\title{
2D-Cellular Automata: Evolution and Boundary Defects
}

\author{
Wani Shah Jahan', Fasel Qadir ${ }^{2}$ \\ ${ }^{1}$ Department of Electronics, S. P. College, Cluster University Srinagar, J\&K, India-190001 \\ ${ }^{2}$ PG Department of Computer Sciences, University of Kashmir, J\&K, India-190006
}

\begin{abstract}
Cellular Automata rules producing evolution type phenomena have been used for a wide range of applications. Various models have been designed and explored for different applications. Although the strength of its parallelism has been felt by various researchers but its exploration for applications will not minimize the hardware but also maximize the optimum strength of processors. Our present study was intended to identify the additive $2 D$ Cellular Automata linear rules on the quality of pattern evolution and the periodic parallelism utilization. We have made an analysis of $2 D C A$ linear game of life (GOL) rule in Neumann neighborhood pattern evolution and observed pattern multiplication in the process. The results achieved will not only minimize the required hardware for parallel channel creation but also expand the microcomputer processing horizon.
\end{abstract}

Keywords: Cellular Automata, Boundary Conditions, Computer Simulation, Patterns Generation;

\section{Introduction}

Von Neumann and Stanislaw Ulam introduced cellular lattice in late $1940 \mathrm{~s}$ as a frame work for modeling complex structures capable of self-reproduction [1]. Cellular Automata is based on a concept of dividing space into a regular lattice structure of cells where each cell can take a set of ' $n$ ' possible values. The value of the cell change in discrete time steps by the application of rule R that depends on the neighborhood around the cell. The neighborhood can be along a line, in a plane or in space. Cellular Automata (CA) model is composed of a universe of cells in a state having neighborhood and local rule. With the advancement of time in discrete steps the cell changes its value in accordance to the state of its neighbors. Thus the rules of the system are local and uniform. There are one- dimensional, two-dimensional and three-dimensional CA models. In onedimensional CA the cells are like a linear canvas and the values of the canvas cells change due to application of a local rule in discrete advancing time steps. In two-dimensional CA the cells form a canvas plane and the changes take place in two dimensions while as in three dimensional $\mathrm{CA}$ volumetric changes take place by the application of local rule with advancement of time. As image is two dimensional data matrix, here we use 2DCA model, where cells are arranged in a two dimensional canvas matrix having interaction with neighboring cells. The central space represents the target cell (cell under consideration) and all spaces around represent its eight nearest neighbors. The structure of the neighbors mostly discussed and applied include Von Neumann neighborhood and Moore neighborhood, are shown in Figure (1). In Von Neumann neighborhood, four cells are positioned at the orthogonal positions of the target cell $\left(\mathrm{a}_{\mathrm{i}, \mathrm{j}}\right)$ while as Moore neighborhood is extension of Neumann structure with additional four cells placed diagonally at the four corner positions. For simplicity Von Neumann neighborhood cells can be termed as orthogonal neighbors and the additional cells by Moore can be called as corner neighbors.

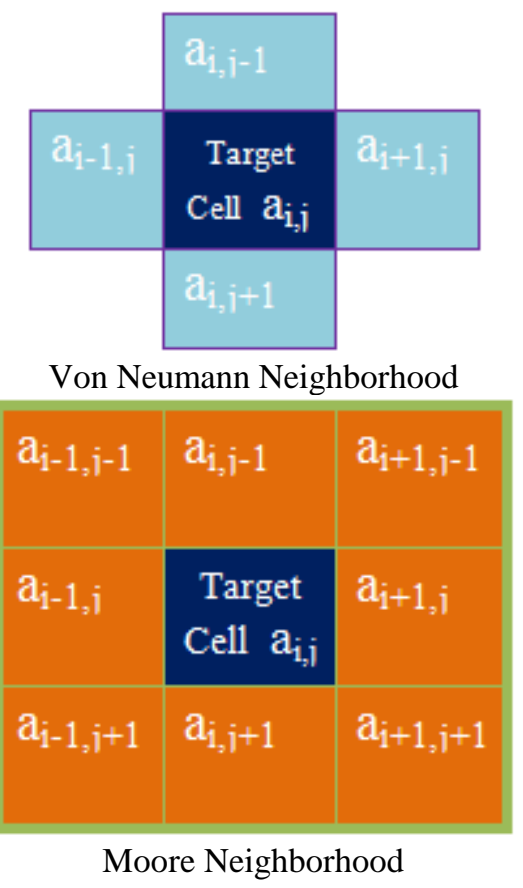

Figure 1

The two dimensional Cellular Automata in general are represented by the equation (I) as given below:

$$
\left[a_{i, j}\right]_{t+1}=R\left[a_{i, j}, a_{i, j+1}, a_{i+1, j}, a_{i, j-1}, a_{i-1, j}\right]_{t}
$$

For Additive Cellular Automata the implementation of the famous totalistic rule in Von Neumann and Moore neighbourhoods, the respective representative equations can be written as follows:

$$
\begin{aligned}
& {\left[a_{i, j}\right]_{t+1}=\operatorname{XOR}\left[a_{i, j}, a_{i, j+1}, a_{i+1, j}, a_{i, j-1}, a_{i-1, j}\right]_{t}} \\
& {\left[a_{i, j}\right]_{t+1}=\operatorname{XOR}\left[a_{i, j}, a_{i-1, j-1}, \ldots \ldots, a_{i+1, j+1}\right]_{t}}
\end{aligned}
$$

Since the exploring worksheet/canvas is practically limited, researchers $[2,3,4,5]$ have defined some boundary conditions to facilitate the protection of data overflow outside the edges 


\section{International Journal of Science and Research (IJSR) \\ ISSN (Online): 2319-7064}

Index Copernicus Value (2015): 78.96 | Impact Factor (2015): 6.391

of the worksheet. On the basis of the applied boundary conditions the cellular automata have been divided into three main categories, briefly defined as follows:

\subsection{Null Boundary Cellular Automata (NBCA)}

Under null boundary conditions the extreme edge cells are having zero values. For 1DCA the extreme right cell and the extreme left cell are considered to be having a value of binary zero ' 0 '

\subsection{Periodic Boundary Cellular Automata (PBCA)}

Under periodic boundary conditions the canvas is considered to be folded so that the extreme cells are taken to be adjacent to each other. For 1DCA the extreme right cell is considered to be adjacent to extreme left cell and the extreme left cell is considered to be adjacent to extreme right cell.

\subsection{Intermediate Boundary Cellular Automata (IBCA)}

Under intermediate boundary conditions the left neighbor of the leftmost cell is regarded as the second right neighbor and right neighbor of the rightmost cell is considered as the second left neighbour.

The linear additive 2-dimensional cellular automata attracted a number of researchers who have applied the rules for various applications in industry and research. The most important among such applications is the VLSI design that uses the cellular automata under the periodic boundary conditions. We have earlier made use of rules under null boundary conditions for cryptography and graphical translations. [8, 9]

The status value of the target cell at time $t+1$ depends on its own status value and the status value of cells in the Moore neighbourhood at time $t$, where $t$ is earlier time than $t+1$. A number of research studies have been carried out by Stephen Wolfram and Norman H. Packard who categorized these rules under the discussed neighbourhoods into general, symmetric and totalistic. The famous example of 9neighborhood totalistic cellular automata is John Horton Conway's 'Game of Life'. Use of the Game of Life rule in Von Neumann neighborhood has also been reported for pattern generation on multiple geometrical shapes and the IR image data analysis by Shah Jahan Wani, Fasel Qadir, Khan K. A. and Peer M. A., [10, 12].

Various studies have also been carried out by Pabitra Pal Choudhury et. el., who classified the cellular automata rules in Moore neighbourhood by assigning the rule values to different cells as shown in Figure (2). The rules are generated by the interaction of target cell with itself and with the 8neighbors around it. These nine rules are said to be basic or fundamental rules and group rules are derived from their combination, Group 2 are rules generated by addition of two basic rules, Group 3 by the combination of three basic rules, Group 4 by the combination of four basic rules, Group 5 by the combination of five basic rules and so on. Group 9 rule (only rule in the group) is the combination of all basic rules. All the combinations are additive (i.e. EX-OR operation).

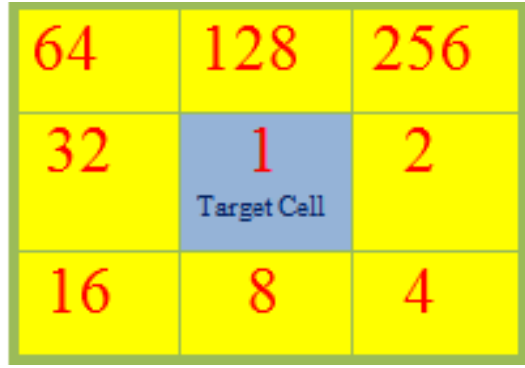

Figure 2

Examples: (Linear CA)

Rule 3 = Rule $2 \oplus$ Rule 1

(Group 2)

Rule $11=$ Rule $8 \oplus$ Rule $2 \oplus$ Rule 1

(Group 3)

\section{Rule $15=$ Rule $8 \oplus$ Rule $4 \oplus$ Rule $2 \oplus$ Rule 1 (Group 4)}

Using this sort of configuration of patterns in 1DCA [6,7], we have reported [10] 2DCA for cryptographic applications where the plaintext is converted to pattern based cipher. The cipher on the receiving end can be converted back to plaintext using the same CA rule in the forward iterations. This technique of generating ciphers has advantages of high cracking immunity due to wide range of rule possibilities and low hardware cost of implementation using VLSI technology.

According to criteria of applying cellular automata rules to a group of data in any neighborhood, the cellular automata have been divided into two types:

i) Uniform Cellular Automata

ii) Non-Uniform Cellular Automata

Uniform $\boldsymbol{C A}$ also known as Linear CA is where rule is applied uniformly on a data matrix of cells. All the cells in matrix get operated with the same rule.

Non-Uniform $\boldsymbol{C A}$ also known as Hybrid CA is one in which all the cells of the matrix have their own local rule that may be different from the rule applied to other cells of that matrix.

\section{Methodology}

For the experimentation purpose, we have used a null matrix of $(129 \times 129)$ elements and binary image of the seed was loaded at its centre. As the CA totalistic rule was applied it started generating patterns. The iteration loop control and the boundary conditions were also applied according to the following algorithm:

Start

Load a Null Matrix

Input Binary Seed

Start Iteration Counter

Label Apply CA Rule

Decrement Iteration Counter

Loop to Label on Counter Condition

Output Resulting Pattern

Stop

Flowchart 


\section{International Journal of Science and Research (IJSR) \\ ISSN (Online): 2319-7064}

Index Copernicus Value (2015): 78.96 | Impact Factor (2015): 6.391

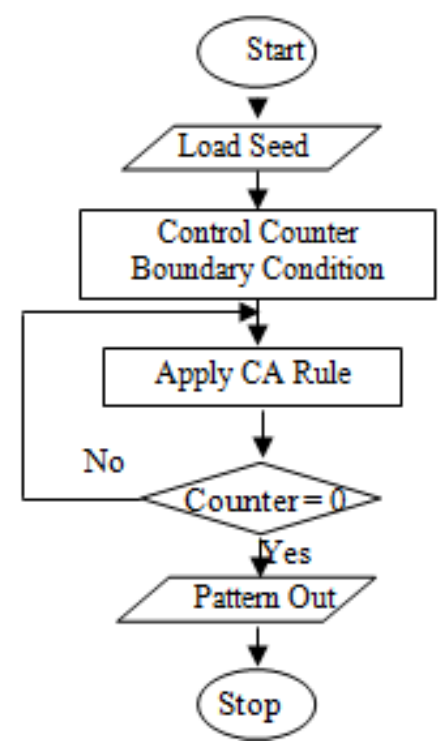

\subsection{Pattern Generation Using Boundary Defects}

In this study we have made the use of above methodology to observe the effect of null boundary on the world of full life i.e. universal matrix of ones. The dark or null boundary is a rectangle of zeros. Some resulting pattern generation references are indicated in Table (1) below:

Table 1: Boundary Defect Patterns

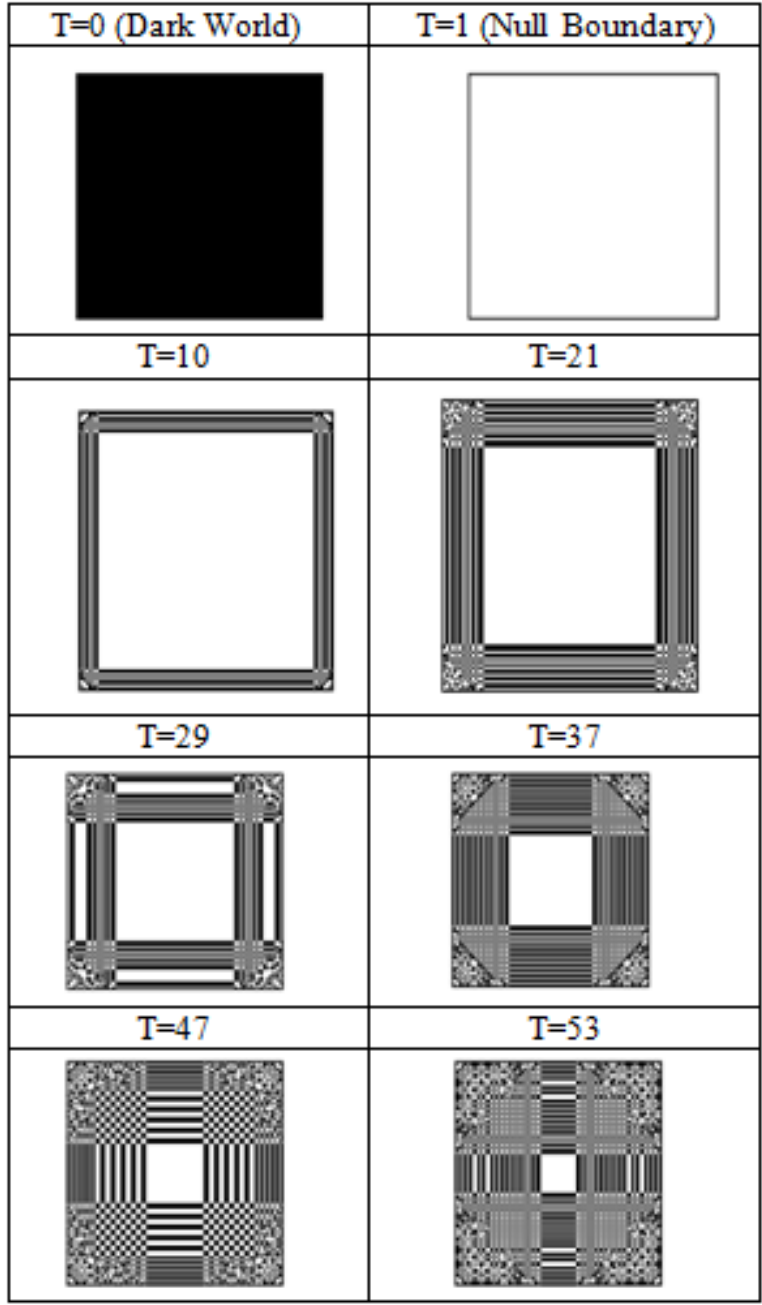

\subsection{Single Seed Boundary Invasion}

\section{Pattern Generation}

We have studied the pattern generation using totalistic cellular automata Game of Life rule (Rule 170) in Neumann neighborhood for the different orientations and have achieved results that could lead to various field applications for interdisciplinary research. In this new exploration we have used various geometrical orientations of the input seed and boundary conditions for the generation of the patterns in evolution as well as what can be termed as boundary invasion. The different orientations used range from simple regular shapes to boundary line matrices and deliberately introduced boundary defects. Different seed shape exploration generated the following pattern results on the MATLAB simulations under null boundary conditions and boundary invading conditions.

In this study same methodology is used to observe the effect of null boundary with a single central live seed on the world of full life i.e. universal matrix of ones. The dark or null boundary is a square of zeros with every side having a central seed of one. Some resulting pattern generation references are indicated in Table (2) below:

Table 2: Single Seed Boundary Defect Patterns

\begin{tabular}{|c|c|}
\hline $\mathrm{T}=0$ (Dark World) & $\mathrm{T}=1$ (Null Boundary) \\
\hline & \\
\hline & \\
& \\
\hline $\mathrm{T}=10$ & $\mathrm{~T}=21$ \\
\hline
\end{tabular}


International Journal of Science and Research (IJSR)

ISSN (Online): 2319-7064

Index Copernicus Value (2015): 78.96 | Impact Factor (2015): 6.391

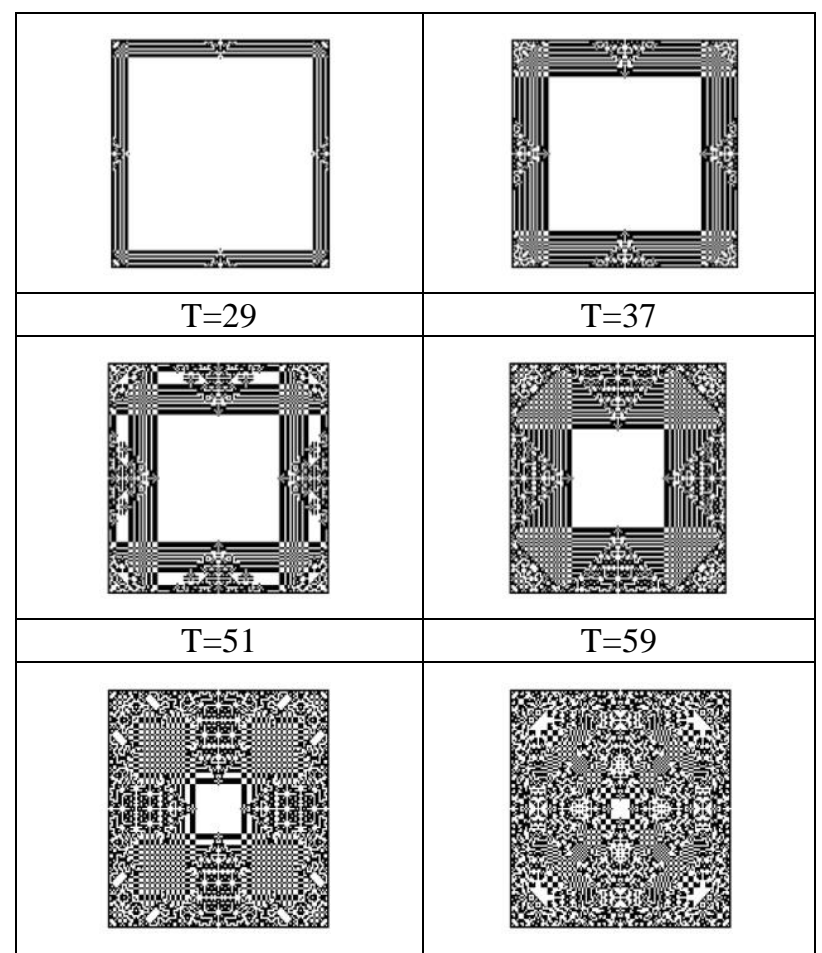

\subsection{Single Seed Evolution and Boundary Invasion}

Moving ahead with the above methodology here we observe the effect of null boundary with a single central live seed at the centre of a world of full life i.e. universal matrix of ones. The dark or null boundary is a square of zeros. A single live seed is introduced at the centre of the matrix. Some resulting pattern generation references are indicated in Table (3) below:

Table 3: Evolution and Boundary Invasion Patterns

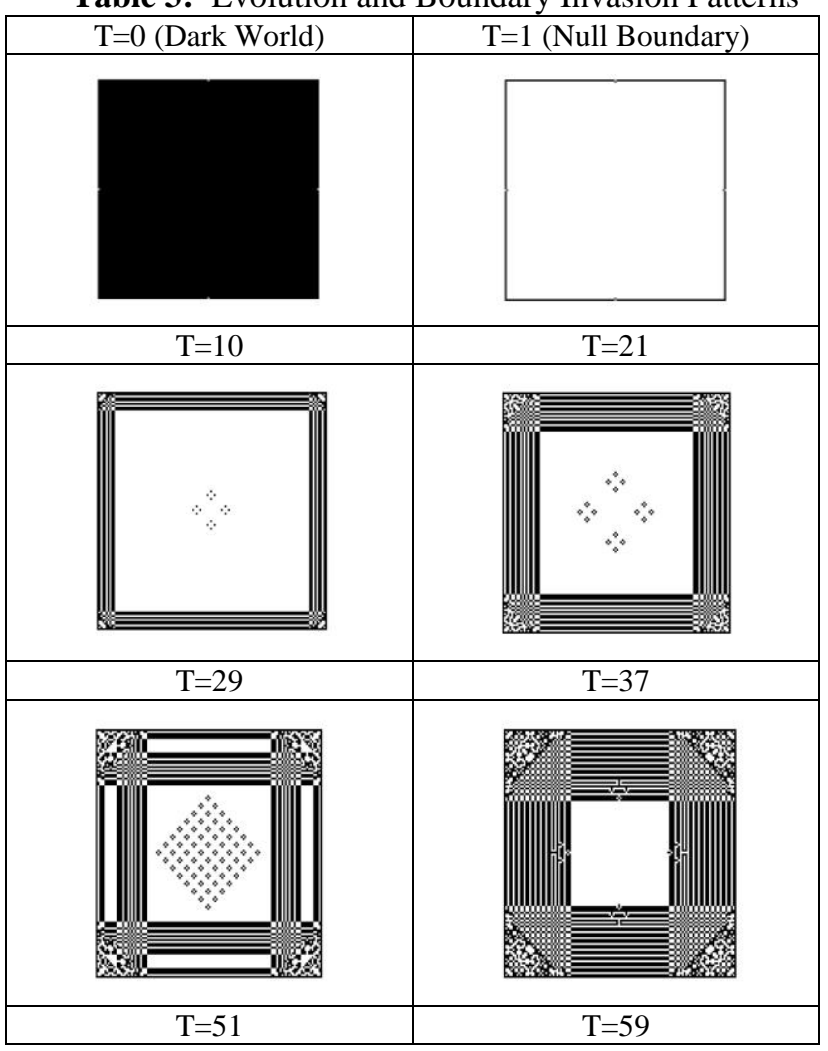

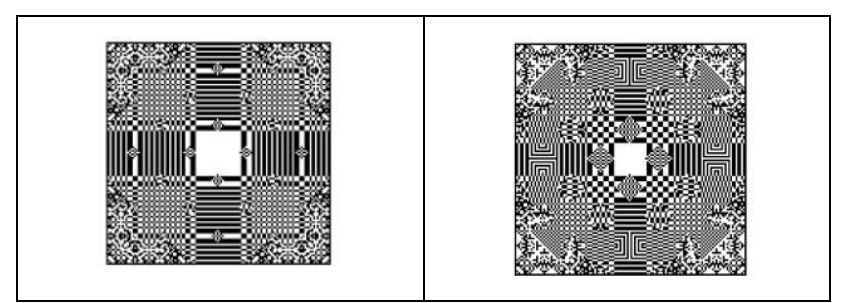

\subsection{Pattern Generation Using Star Shaped Data Block}

Here we introduce a multi seed data block with the above methodology to observe the effect of evolution with the application of same rule. The evolution results in a periodic multiplication of the data block in various orientations. Some resulting pattern generation references are indicated in Table (4) below:

Table 4: Multi Seed Evolution Patterns

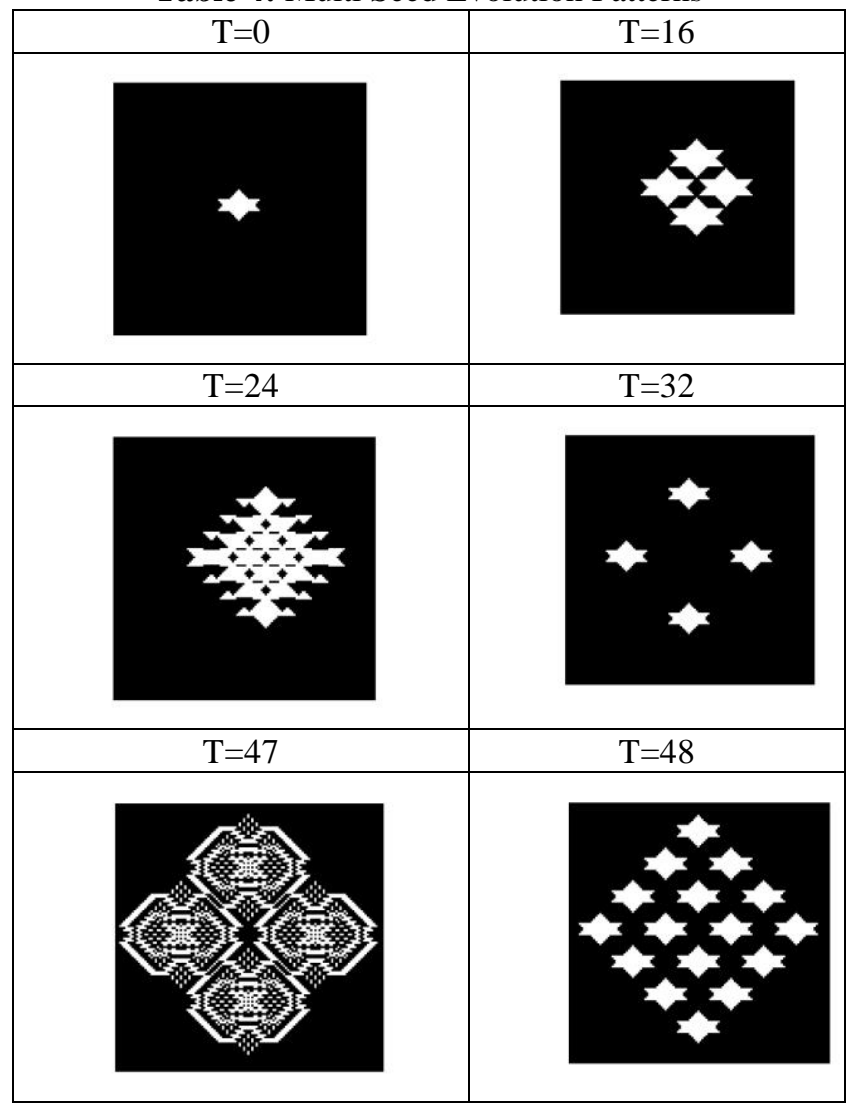

The periodicity of the recurrence after fixed 16 iterations illuminates a path towards the use of linear CA rules for packet data cryptography and image watermarking applications. Second important periodicity is the multiplication and scattering of cluster shapes after fixed intervals that can applied for the simulation of physical systems.

\section{Discussion and Conclusion}

Pattern generation as well as single seed evolution has been reported by Neumann and Ulam in 1950's and later on by various other authors in one dimension, and a few in two dimensions as well, but the case of boundary interaction in the domain of cellular field has not been reported by any author. In one dimensional Cellular Automata Stephen 
Wolfram has authored a comprehensive study on the subject and touched the boundary interactions in detail but the pattern generation with this type of phenomena has not been touched. Other important finding in this work is the evolution patterns of multiple seed cluster shapes. These outcomes here seem to be analogous to radio energy wave front patterns. The results are definitely not only showing a bidirectional interaction that can be useful for studying physical processes under environmental conditions but also a powerful parallelism. The power of the parallelism can be utilized to minimize the hardware complexities for secured cluster data communications.

\section{References}

[1] J. Von Neumann. Theory of Self-Reproducing Automata: University of Illinois Press, 1966.

[2] Norman H. Packard and S. Wolfram. Two Dimensional cellular Automata: J. Stat. Phys. 1986, Vol. 38.

[3] S. Wolfram., Twenty Problems in the Theory of CA, Physica Scripta, 1985, pp. 170-183, Vol T9.

[4] Jarkko Kari. Universal Pattern Generation with Cellular Automata, Theoretical Computer Science, Vol. 429, April, 2012

[5] Choudhury P. P et al. Classification of CA Rules based on their Properties., IJCC Dec. 2010.

[6] Makoto Itoh and Leon O. Chua, "Difference Equations for Cellular Automata", International Journal of Bifurcation and Chaos, Vol. 19 No.3 (2009) 805-830.

[7] Radu V.Craiu and Thomas C. M. Lee "Pattern Geeration Using Likelihood Interference for Cellular Automata" IEEE Transactions on Image Processing Vol. 15 No. 7, July 2006.

[8] Wani Shah Jahan, Khan K. A. and Peer M. A. "2DCellular Automata Linear Rules for Cryptography Based on Pattern Evolution" International Journal of Advanced Research in Computer Science Engineering and Information Technology vol 2 no. 3, April, 2014.

[9] Fasel Qadir, Shah Jahan, Peer M. A. and Khan K. A. "Replacement of Graphic Translations with TwoDimensional Cellular Automata, Twenty Five Neighborhood Model" IJCEM July 2012, 33-39.

[10] Shah Jahan Wani, Fasel Qadir, Khan K. A. and Peer M. A., (2013, Dec.) Springer Conference ICICIC Global "Pattern Generation Using 2D Cellular Automata on Multiplr Geometrical Shapes"

[11]Fasel Qadir, P. Z. Ahmad, Wani S. J., Peer M. A. , Quantum-Dot Cellular Automata: Theory and Applications, IEEE Conference on Machine Intelligence and Research Advancement (ICMIRA-2013), December-2013, 540-544.

[12] Shahi Jahan Wani and Fasel Qadir, "Cellular Automata Based Study of Spectral Signatures of Dal Lake Infrared Imagery", Oriental Journal of Computer Science and Technology, Vol. 10, No. 2, June 2017. 